\title{
Creating an experimental testbed for information-theoretic analysis of architectures for x-ray anomaly detection
}

David Coccarelli, Joel A. Greenberg, Sagar Mandava, Qian Gong, Liang-Chih Huang, et al. 


\title{
Creating an Experimental Testbed for Information-Theoretic Analysis of Architectures for X-ray Anomaly Detection
}

\author{
David Coccarellia , Joel A. Greenberga ${ }^{\mathrm{a}}$, Sagar Mandava ${ }^{\mathrm{b}}$, Qian Gong ${ }^{\mathrm{a}}$, Liang-Chih Huang ${ }^{\mathrm{c}}$, \\ Amit Ashok ${ }^{\mathrm{c}}$, and Michael E. Gehm ${ }^{\mathrm{a}}$ \\ ${ }^{a}$ ECE Department, Duke University, Durham, NC, USA \\ ${ }^{\mathrm{b}}$ ECE Department, University of Arizona, Tucson, AZ, USA \\ ${ }^{\mathrm{c}}$ College of Optical Sciences, University of Arizona, Tucson, AZ, USA
}

\begin{abstract}
Anomaly detection requires a system that can reliably convert measurements of an object into knowledge about that object. Previously, we have shown that an information-theoretic approach to the design and analysis of such systems provides insight into system performance as it pertains to architectural variations in source fluence, view number/angle, spectral resolution, and spatial resolution. ${ }^{1}$ However, this work was based on simulated measurements which, in turn, relied on assumptions made in our simulation models and virtual objects.

In this work, we describe our experimental testbed capable of making transmission x-ray measurements. The spatial, spectral, and temporal resolution is sufficient to validate aspects of the simulation-based framework, including the forward models, bag packing techniques, and performance analysis. In our experimental CT system, designed baggage is placed on a rotation stage located between a tungsten-anode source and a spectroscopic detector array. The setup is able to measure a full $360^{\circ}$ rotation with 18,000 views, each of which defines a $10 \mathrm{~ms}$ exposure of 1,536 detector elements, each with 64 spectral channels. Measurements were made of 1,000 bags that comprise 100 clutter instantiations each with 10 different target materials. Moreover, we develop a systematic way to generate bags representative of our desired clutter and target distributions. This gives the dataset a statistical significance valuable in future investigations.
\end{abstract}

Keywords: Information Theory, High Dimensionality, X-Ray System Geometry, X-Ray System Architecture

\section{INTRODUCTION}

The problem of concealed threat detection in baggage is important to aviation security. ${ }^{2}$ Governments and security equipment vendors both want to know how to design machines and quantify how well they perform. Since many detection applications are focused on performing a specific task, and image quality is often objectdependent, the choice of test objects is critical to the overall analysis approach and impacts the conclusions drawn. Therefore, to quantitatively analyze system performance, one needs a principled way of creating bags relevant to the security space and also making measurements. Unfortunately, most approaches to date lack a rigorous justification of their methods and, to our knowledge, there is no database of x-ray measurements which takes the care to sample a target object space with a principled approach.

In this paper, we describe our approach to the creation of a bag ensemble that is relevant to the detection of concealed objects in luggage. We demonstrate this approach experimentally by creating over 1000 bags, performing planar scans of each in a home-built spectral CT system, and organizing the resulting data into a database that is freely available. This approach and the availability of the data will aid in the develop of next-generation scanner technology and detection algorithms.

Our use of energy sensitive detectors can give better insight to the material specific information about the items being scanned. ${ }^{3,4}$ Because the information is material specific, it can be important to the task of differentiating materials and relevant to threat detection in the security space. Moreover, it has been shown

Further author information: (Send correspondence to David Coccarelli)

David Coccarelli: E-mail: david.coccarelli@duke.edu

Anomaly Detection and Imaging with X-Rays (ADIX) II, edited by Amit Ashok, Edward D. Franco,

Michael E. Gehm, Mark A. Neifeld, Proc. of SPIE Vol. 10187, 1018709 · @ 2017 SPIE

CCC code: $0277-786 \mathrm{X} / 17 / \$ 18 \cdot$ doi: $10.1117 / 12.2263033$ 
that the photon counting and energy resolving detectors can be useful in the identification of target materials when used in the CT system. ${ }^{5-8}$ Most commonly, material segmentation in the medical space has focused on the use of dual-energy systems. ${ }^{9}$ However, it has been shown that the two-dimensional basis that this approach assumes may not be sufficient to categorize a wider class of materials. ${ }^{10}$ By creating measurements with 64 energy channels, we can provide more information for the classification task.

\section{BAGGAGE ENSEMBLES}

We create an ensemble composed of over 100 items that include both stream of commerce objects and threat/nonthreat pairs. The stream of commerce items will be drawn from 4 categories (basics, clothes, electronics, and health/toiletries) with relevant proportions. To ensure that the bags contain random object distributions (both in terms of object selection and location/orientation) that are consistent with the prescribed probabilities, we developed a bag packing algorithm that specifies the bag contents and configuration. Once we have packed the bag, we generate a text-based bag ground truth by recording the location, orientation and item identification number of all included items. This ground truth meta-data is a critical element in the overall analysis chain and an element that is missing in conventional data sets.

In order to take data useful to detection and classification tasks, we introduce two classes of materials that are strategically placed in the bags. We use a threat class to designate potential security risks and a non-threat class for benign materials. Each threat material is paired with a non-threat material chosen to be very similar to each other so that any classifier designed from the data may straddle the decision boundary. The threat/non-threat pairs are based on material alone; there are no shape-based threats. Because densities $(\rho)$ and effective atomic numbers $\left(Z_{\text {eff }}\right)$ are two material characteristics that determine x-ray transmission behavior, the threat and non-threat items are chosen to have very similar $\rho$ and $Z_{\text {eff }}$. In each pair, the materials are placed in identical containers with identical sizes, shapes, locations and placements within the bag. This allows the material to be the only factor differentiating two bag scans within a material pair. An example of one of these pairs is olive oil (non-threat) and acetone (threat). Olive oil has a $Z_{\text {eff }}$ of about 6 with a $\rho$ of $0.9 \mathrm{~g} / \mathrm{cm}^{3}$ and acetone has a $Z_{\text {eff }}$ of about 6.5 with a $\rho$ of $0.8 \mathrm{~g} / \mathrm{cm}^{3}$. We choose 5 such threat/non-threat pairs and measure 100 threat/100 non-threat bags for each pair. A total of 500 non-threat and 500 threat bags are measured with each threat/non-threat target being placed in each clutter instantiation.

\section{EXPERIMENTAL SETUP}

\subsection{Approach}

Using a single X-ray source, we illuminate a designed bag consisting of a variety of objects. The bag is placed and rotated on a rotation stage to enable the acquisition of projection images from a multitude of angles. The transmitted X-rays are recorded using a linear array of energy-sensitive detector pixels at high spatial, spectral, and temporal resolution. We can bin this data to create subsets with differing incident flux, spatial resolution, angular/view sampling, and spectral bin size. The detector array consists of 6 MulitX detector modules, each 128 pixels covering $10 \mathrm{~cm}$. The array is translated to two positions in order to cover a total of $1.25 \mathrm{~m}$, including minor spacing between modules. A schematic of the system and summary of the system parameters are shown below in Fig. 1 and Table 1, respectively.

\begin{tabular}{|l|l|}
\hline Parameter & Value \\
\hline Source & Tungsten Anode at $140 \mathrm{kVp}$ and $0.35 \mathrm{~mA}$ (SANDT) \\
Spectral Resolution & 64 channels $20-170 \mathrm{kV}$ \\
Spatial Resolution & 1536 pixels at $800 \mu \mathrm{m} \times 800 \mu \mathrm{m}$ \\
Angular Resolution & 18,000 views over $360^{\circ}$ \\
Temporal Resolution & $10 \mathrm{~ms}$ per view \\
View Angle Resolution & $0.02^{\circ}$ \\
\hline \multicolumn{2}{|c|}{ Table 1. The experimental parameters used during data collection. }
\end{tabular}



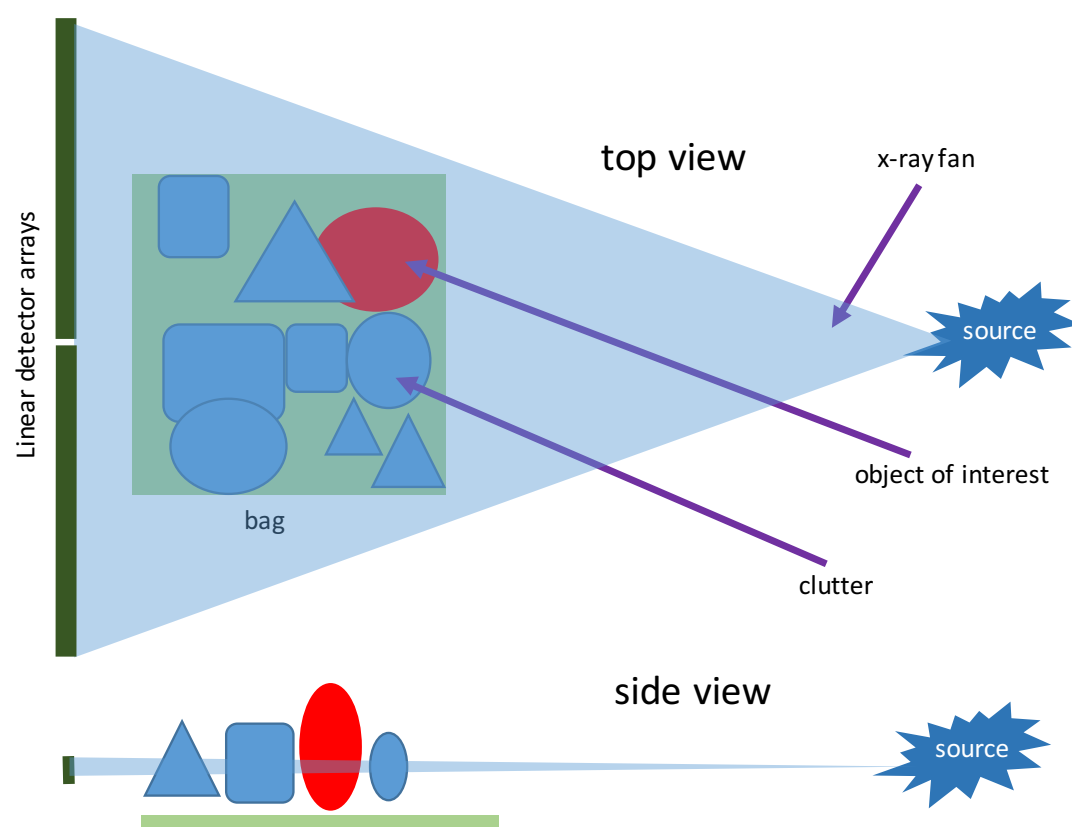

Figure 1. The image shows an overhead view experimental setup in which a stationary source illuminates a bag, which rotates through a full $360^{\circ}$. The transmitted x-rays are detected by a linear array of energy sensitive detector pixels.

The bag is rotated at a speed of $2^{\circ}$ per second. Counts on the detector are acquired in consecutive $10 \mathrm{~ms}$ windows. When the system is rotated over a full rotation $\left(360^{\circ}\right)$, this results in data with 18,000 corresponding views angles. It takes approximately 140 hours of scan time to acquire the data for 1,000 bags. Preparation of the bag, data transfer time, setup, and processing lengthen total data collection time. At full spatial, temporal, and spectral resolution, the data set for the entire experiment, 1,000 bags, is approximately $3.5 \mathrm{~TB}$.

\subsection{Hardware}

The hardware involved in the testbed consists of an x-ray source, bag staging pedestal, and detector array. The entire setup can be seen in Fig. 2. The x-ray tube is located on the far right of the figure. It is manufactured by the SANDT Corporation, model SAXG1701, and uses a tungsten anode. The tube can be seen in Fig. 3. It emits x-rays towards the bag area which interact with the materials packed in the bag. The attenuated x-rays are then measured at the detector array placed on a translation stage behind the bag. 


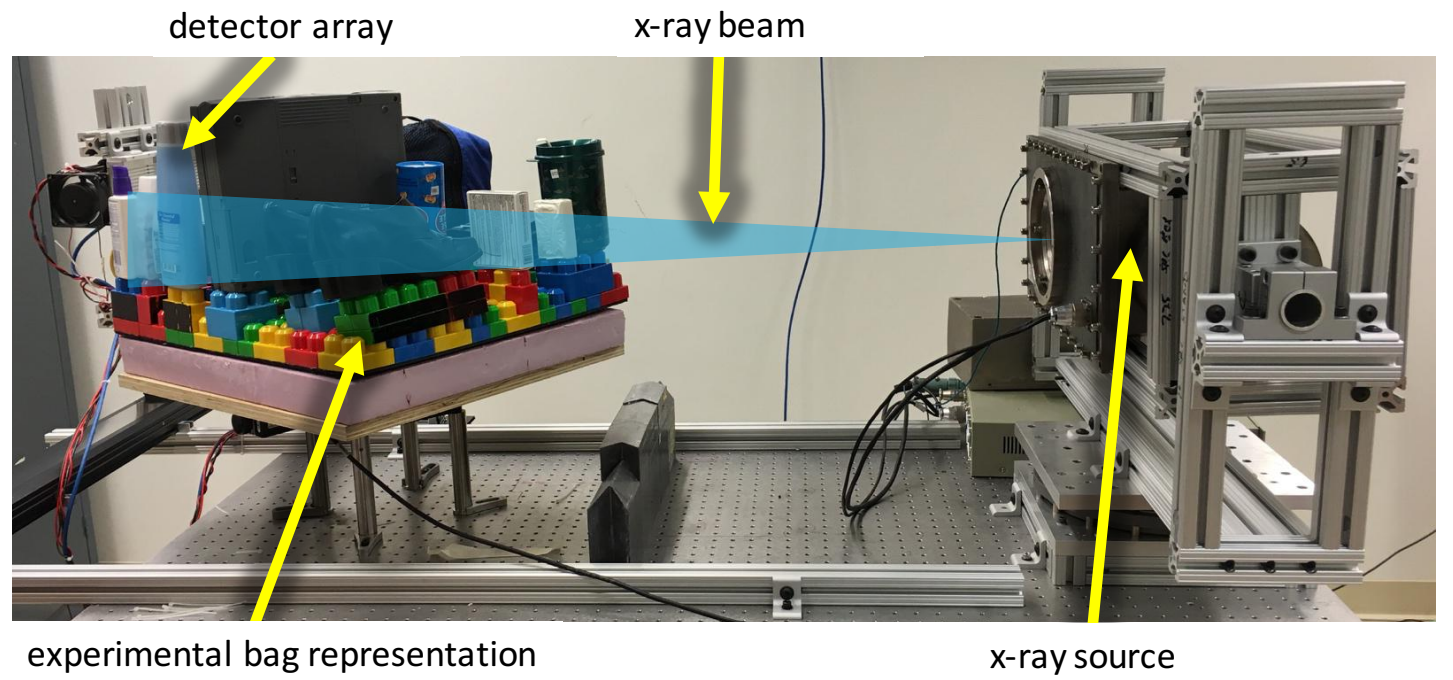

Figure 2. The experimental setup is shown. The x-ray source is mounted to an optical table on the right of the image. It produces radiation that is attenuated by the experimental bag representation and measured at the detector array. The $\mathrm{x}$-ray beam shape represents the location of relevant primary rays that are attenuated by the materials in the bag.

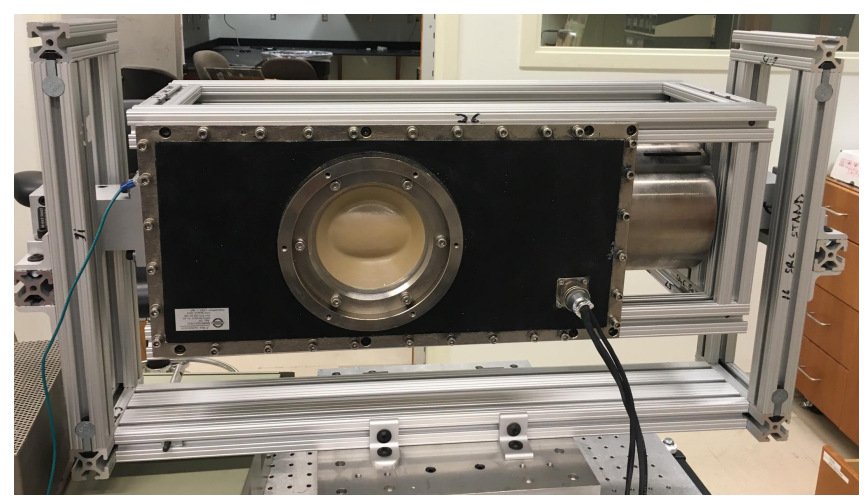

Figure 3. The $1.2 \mathrm{~mm}$ focal-spot tungsten anode tube (SANDT SAXG1701) is shown mounted in a 80/20 structure that allows for its alignment. The window of the tube faces outward, and no filtering is applied except for that native to the tube.

The rotation stage is controlled by a Newport Universal Motion Controller/Driver, Model ESP300. The inclusion of the rotation stage can be seen in both Fig. 2 and Fig. 4. The stage is controlled from custom software that interacts with the controller though publicly the available Newport API. To mitigate x-ray scattering effects from the dense rotation stage, the packed bag is elevated from the rotation stage using a layer of foam. This material helps to isolate the bag location from the stage and optical table. 


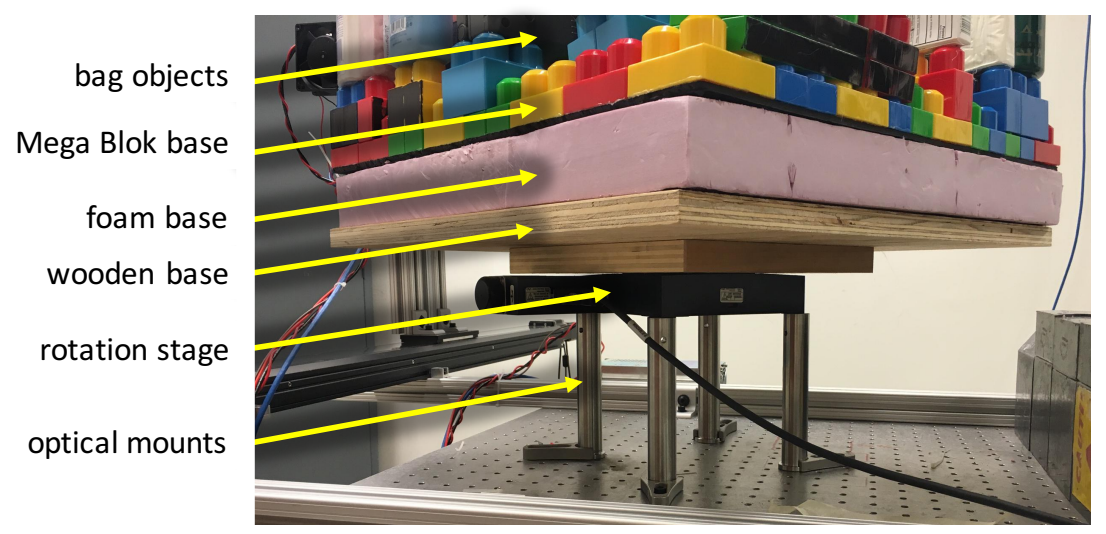

Figure 4. The structure of the bag pedestal is shown. The bag items are placed on a block grid that make up the Mega Blok base. This grid is placed on a foundation of foam to elevate the measured materials from the denser, more scattering materials closer to the table. A wooden base is used to support the foam base and affix it to the rotation stage. The rotation stage is firmly mounted to the optical table after alignment.

The detector array is composed of 6 MultiX detector modules as seen in Fig. 5. Each detector module has $128800 \mu \mathrm{m} \times 800 \mu \mathrm{m}$ pixels arranged in a line. The 6 detector modules are aligned so that a row of 786 pixels is formed. This array is mounted to a translation stage that is controlled by custom software. The stage moves the array to its two locations during acquisition. The detector response is characterized by a pixel-level gain and energy measurement seen in Fig. 6. One sees the pixel-to-pixel variation along the horizontal axis and the energy-dependent response along the vertical axis. By taking this acquisition without obstruction from items in the beam path, we create data useful for normalizing the measurements made of the designed baggage.

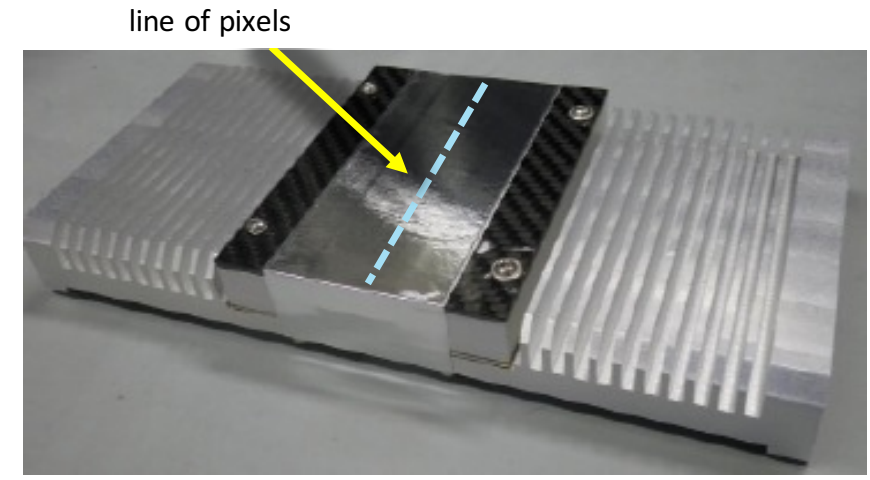

(a)

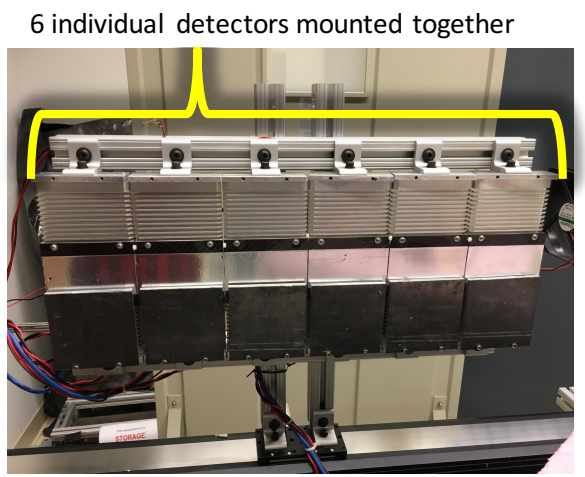

(b)

Figure 5. A single detector module, 5(a), and the detector array, 5(b) are shown. The detector module has a line of 128 detector pixels that lies underneath the metallic tape at its center. To form the detector array, 6 modules are mounted to an 80/20 frame that is then attached to a Zaber translation stage. Wiring behind the modules allow for communication and control, while fans help to regulate the temperature of the electronics. 


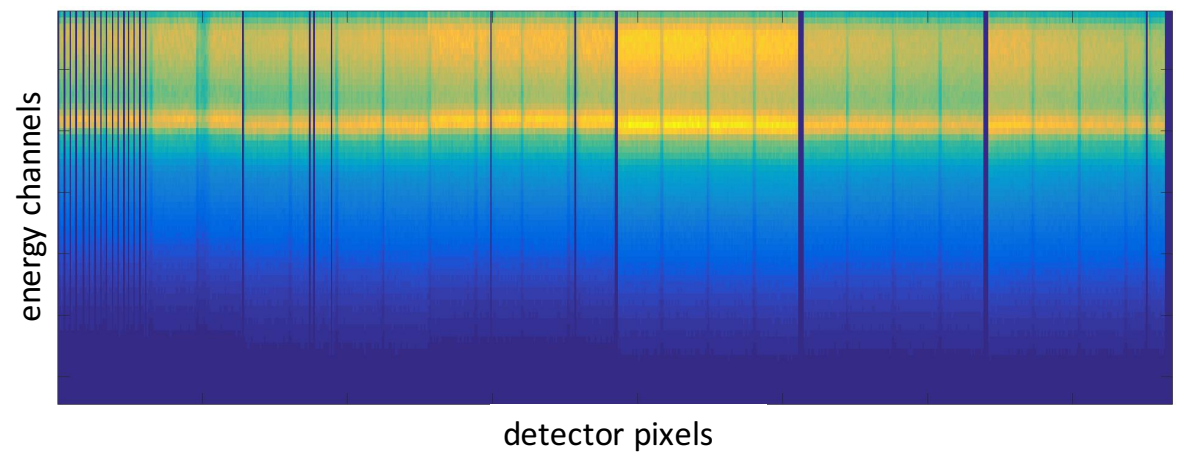

Figure 6. The detector response from a consistent x-ray exposure (color available in online version). Yellow signal corresponds to higher counts, and blue lower counts. The detector array was moved so that each detector pixel was illuminated at the same location, eliminating inconsistencies from the structure of the beam. This highlights the pixelto-pixel variation as well as the spectra of the source. Differences are seen in the intensities across pixels which show underperforming locations such as dim vertical lines or dead pixels as dark blue vertical lines. Also seen is the spectral from the source in which the characteristic tungsten peak shows up as a yellow line across detector pixels.

\subsection{Acquisition and Quality Control}

To ensure that the system is efficient and repeatable, we develop software to automate the motion of the bag and detectors as well as the detector readout and storage. To ensure quality control throughout the measurements, we periodically acquire data sets with no bag present as a reference. Furthermore, we include a Delrin rod as a fiducial marker. It is used as a reference in each data set. The location of this fiducial marker is checked in each scan to ensure proper system alignment and component location. If the location of the fiducial is incorrect, the scan can be reacquired and the system checked.

The proper alignment and characterization of the system is crucial to clear image reconstructions. We use a simple filtered back projection algorithm to reconstruct initial images as a means to measure our progress as we align and characterize the system. We achieve rough system alignment with the use of laser levels and laser distance measure. By reconstructing simple, known objects and evaluating the image artifacts and/or errors, we iteratively modify the experimental configuration to match well the idealized FBP model. Final characterization can then be made through updates to the parameters that describe the system. For the filtered back projection approach, this involves the exact spatial location of the detector pixels, center of rotation, source origin, detector response.

\section{DATA AND REPRESENTATION}

The data acquired by our system consists of $\mathrm{N}$ temporal frames during the object rotation. The projection measurement at each frame is a measurement of the number of photons arriving at each detector pixel within each of 64 energy bins. This data is commonly displayed as a sinogram. A diagram where the data from each view is shown next to subsequent views. An example of a sinogram from a plastic bottle filled with water placed away from the center of rotation. This simple object can be seen in Fig. 7. Each column of the image shows the counts present on the linear detector array. Along the horizontal axis, one can see the progression of the views during acquisition. The yellow pattern represents the attenuation from objects in the beam path, and the sinusoidal pattern is indicative of the rotation of the scanned objects. Because the data is acquired from two detector array locations, the measurements are stitched together so that the top half of the sinogram is acquired, then after the detector movement, the bottom half is acquired.

In order to normalize the data, we divide the measurement by that of a scan without a bag present. This allows for flat fielding of the source signal as well as compensating for pixel-to-pixel inconsistencies and variations in energy-specific pixel responses. Pixel-by-pixel gain maps were also made by exposing each detector pixel to the same location in the source illumination the result of which is seen in Fig. 6. These maps can be used in the processing of raw data to balance nonuniform detector response across pixels and energies. 


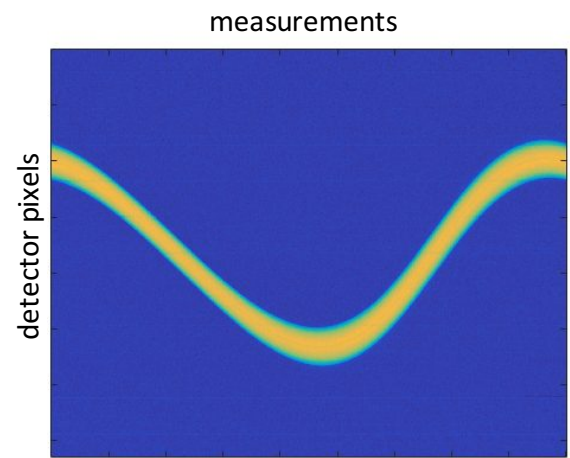

angular views

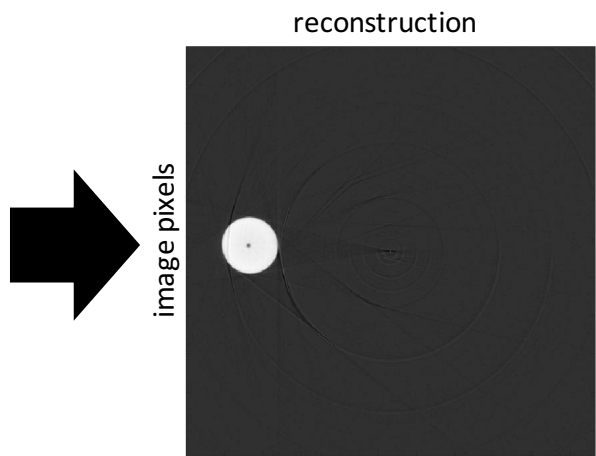

image pixels

Figure 7. The system captures measurements which are displayed as a sinogram in the left part of the figure. The sinogram and image shown correspond to signal after summing counts at all energies. Through reconstruction techniques, these measurements can be transformed to images as seen in the right part of the figure. Here, a single water bottle with a straw was place in a bag and measured. Its sinogram can be seen as a sinusoidal signal on the left and image reconstruction using filtered back projection on the right.

When reconstructing data from a packed bag, one can reconstruct images such as the one in Fig. 8(b). This image shows the reconstruction of the objects to form an image corresponding to the photograph taken after the bag packing, seen in Fig. 8(a). As an example of an easily recognizable object, one can see the coffee mug in the bottom left hand corner of both images. The item is seen as a white mug in Fig. 8(a) and a white circle in Fig. 8(b). The photograph of the packed bag shows the clutter instantiation for 10 scans. Each of these scans includes a different threat/non-threat material. In the case of the reconstruction in Fig. 8(b), the filled circle to the bottom left is a mayonnaise jar filled with methanol. This space is left blank in Fig. 8(a) as it only represents the clutter.

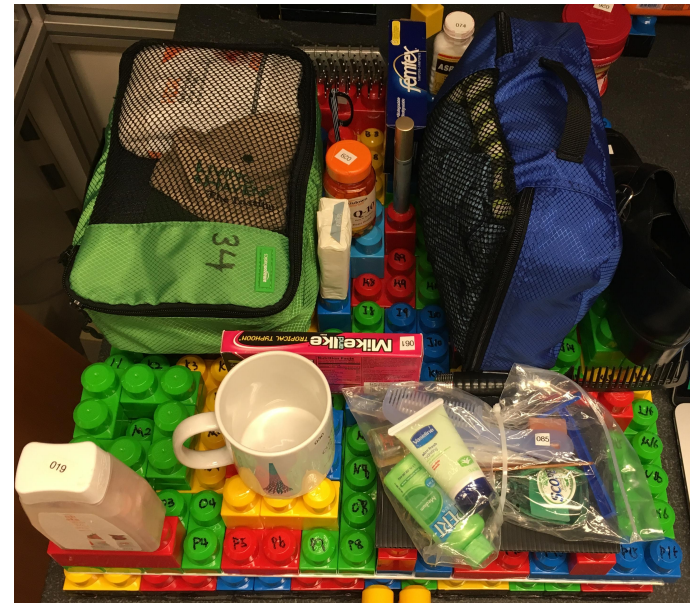

(a)

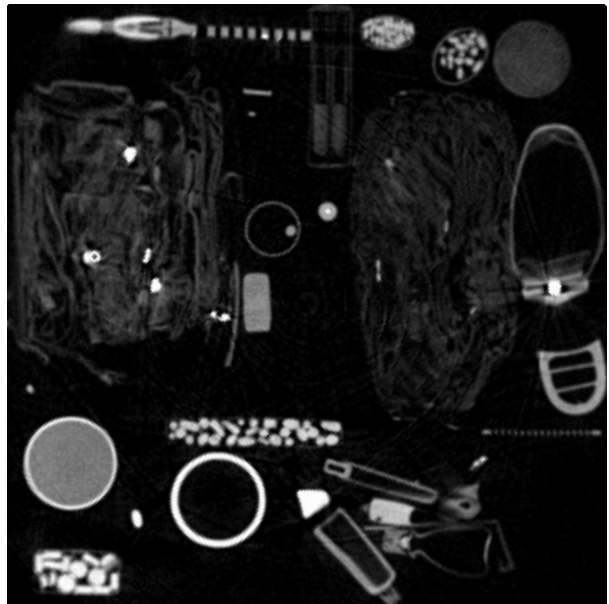

(b)

Figure 8. These figures show an example of a packed bag and a possible reconstruction of its scanned measurements. Figure (a) shows the clutter packed in bags 41 through bag 50. One can see how this clutter matches up with the items in the reconstruction of the data in Figure (b), which is an instance of this clutter with a jar filled with methanol placed in the bottom left of the pictured grid. The reconstruction is performed with a relatively simple filtered back projection algorithm after counts at all energies are summed. Yet it shows the potential of the measurements to yield high quality reconstructed images.

Another feature of the database is that there are 64 energy channels. This means that one can selectively use 
energy channels or combine them in a desired way. Figure 9 shows how this can be helpful when dealing with certain image artifacts. For example, beam hardening artifacts from attenuation with dense metals may be less noticeable at higher energies.

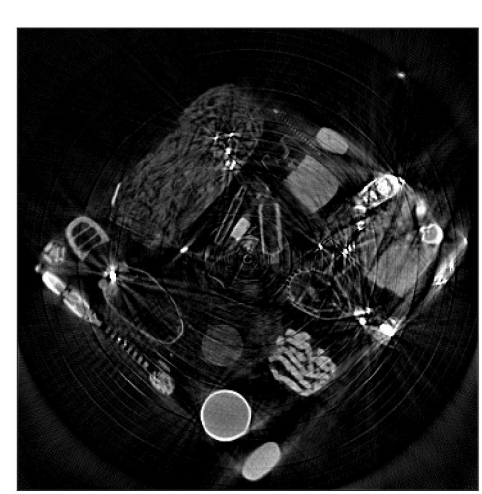

$22.1 \mathrm{kV}-28.7 \mathrm{kV}$

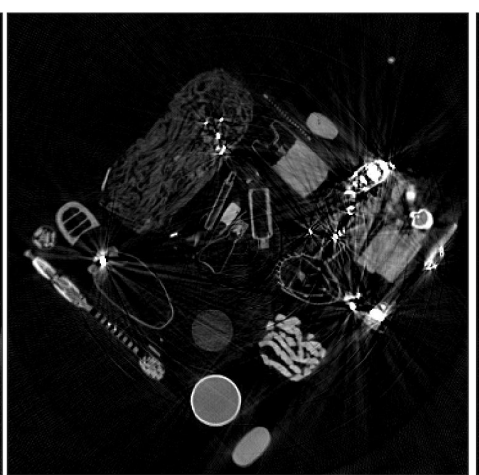

$57.3 \mathrm{kV}-72.7 \mathrm{kV}$

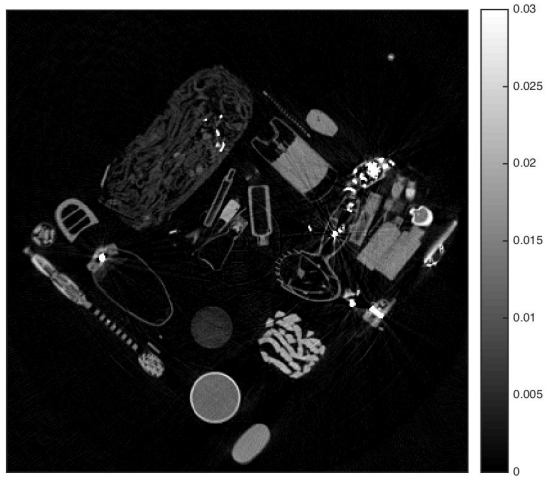

$110.1 \mathrm{kV}-125.5 \mathrm{kV}$

Figure 9. Three reconstructions are shown of the same bag. For each reconstruction, a different energy window was used. Because the data has 64 energy channels, one can select whichever subset of those energies is most beneficial. In the case of these reconstructions, we see that the lower energy measurements are dominated by beam hardening artifacts from higher density materials in the bag. Using a the data for the higher energy photons allows for mitigation of some of these artifacts. This can be seen as the image becomes clearer from the left to right in subsequent images.

\section{CONCLUSIONS}

We present a database of x-ray measurements that can be used for a variety of investigations. The designed nature of the bags scanned gives the data an advantage when used in development of classification techniques as the sample distributions are better sampled with the given number of bag instantiations. The high dimensionality and relevance of the bags and the variable clutter instantiations allows for testing of algorithms that require large amounts of data. In addition, the high spatial and spectral fidelity of the measurements allows for the testing of interesting image reconstruction techniques. The database gives a set of measurements that is ripe for investigation and further discovery in the field of x-ray imaging and threat detection.

\section{ACKNOWLEDGMENTS}

The authors gratefully acknowledge the support of the US Department of Homeland Security through the Advanced X-Ray Material Discrimination Program.

\section{REFERENCES}

[1] Lin, Y., Allouche, G. G., Huang, J., Ashok, A., Gong, Q., Coccarelli, D., Stoian, R.-I., and Gehm, M. E., "Information-theoretic analysis of x-ray photoabsorption based threat detection system for check-point," in [SPIE Defense+ Security], 98470F-98470F, International Society for Optics and Photonics (2016).

[2] Singh, S. and Singh, M., "Explosives detection systems (eds) for aviation security," Signal Processing 83(1), $31-55$ (2003).

[3] Feuerlein, S., Roessl, E., Proksa, R., Martens, G., Klass, O., Jeltsch, M., Rasche, V., Brambs, H.-J., Hoffmann, M. H., and Schlomka, J.-P., "Multienergy photon-counting k-edge imaging: Potential for improved luminal depiction in vascular imaging 1," Radiology 249(3), 1010-1016 (2008).

[4] Macovski, A., Alvarez, R., Chan, J.-H., Stonestrom, J., and Zatz, L., "Energy dependent reconstruction in x-ray computerized tomography," Computers in biology and medicine 6(4), 325IN7335-334336 (1976). 
[5] Schlomka, J., Roessl, E., Dorscheid, R., Dill, S., Martens, G., Istel, T., Bäumer, C., Herrmann, C., Steadman, R., Zeitler, G., et al., "Experimental feasibility of multi-energy photon-counting k-edge imaging in preclinical computed tomography," Physics in medicine and biology 53(15), 4031 (2008).

[6] Wang, X., Meier, D., Mikkelsen, S., Maehlum, G., Wagenaar, D., Tsui, B., Patt, B., and Frey, E., "Microct with energy-resolved photon-counting detectors," Physics in medicine and biology 56(9), 2791 (2011).

[7] Frey, E., Wang, X., Du, Y., Taguchi, K., Xu, J., and Tsui, B., "Investigation of the use of photon counting x-ray detectors with energy discrimination capability for material decomposition in micro-computed tomography," in [Medical Imaging], 65100A-65100A, International Society for Optics and Photonics (2007).

[8] Roessl, E. and Proksa, R., "K-edge imaging in x-ray computed tomography using multi-bin photon counting detectors," Physics in medicine and biology 52(15), 4679 (2007).

[9] Fessler, J. A., Elbakri, I. A., Sukovic, P., and Clinthorne, N. H., "Maximum-likelihood dual-energy tomographic image reconstruction," in [Medical Imaging 2002], 38-49, International Society for Optics and Photonics (2002).

[10] Eger, L., Do, S., Ishwar, P., Karl, W. C., and Pien, H., "A learning-based approach to explosives detection using multi-energy x-ray computed tomography," in [2011 IEEE International Conference on Acoustics, Speech and Signal Processing (ICASSP)], 2004-2007, IEEE (2011). 